\title{
COLLABORATIVE SUPPORT SERVICES FOR CIVIL SERVANTS
}

\author{
Nikolaos V. Karadimas \\ Vassili Loumos \\ National Technical University of Athens, \\ Multimedia Technology Laboratory \\ 9 Heroon Polytechneiou, Zografou Campus, \\ 15780 Athens, Greece \\ E-mail: nkaradimas@medialab.ntua.gr, \\ loumos@cs.ntua.gr
}

\author{
Nikolaos P. Papastamatiou \\ Omega Technology \\ 4 El. Venizelou, Kallithea \\ 17676 Athens, Greece \\ E-mail:nikos@omegatech.gr
}

\section{KEYWORDS}

Collaboration Platforms, Web Services, Social Services, Grid Architecture.

\begin{abstract}
Civil servants, and especially those engaged in social services, have similar needs all over Europe to access and elaborate information on training and employment offers in order to deliver the best possible service to those who have the right. This work extends the Web Services that collect and offer up-to-date information on training and job offers from different web sources matched to the needs of each case (Karadimas and Papastamatiou 2008) to address the Grid architecture. Grid architecture can be defined as a layer of secure networked services that allow users single sign-on access to a distributed collection of data resources, offering up-to-date information on training and job offers from different web resources. The proposed architecture, as it will include personal data that must be secured from unauthorized access, will be based on the Open Grid Services Architecture (OGSA) that defines standard mechanisms for creating, naming, and discovering transient Grid service instances.
\end{abstract}

\section{INTRODUCTION}

The current work proposes the development of an integrated platform based on the Grid/Web Services architecture that will collect and offer up-to-date information on training and job offers from different web sources matched to the needs of beneficiaries (people of disandantaged citizens' groups searching for work and reinforcement). The system will help civil servants to connect the needs of those who have the right.to employment requests and training opportunities combining different web resources on an individual basis, minimizing at the same time communication expenses, data replication and expiration.

This communication will take place through a Grid/Web service installed in each Social Service
Agency that will partially access its database elaborating data about beneficiaries (anonymously) in order to be able to suggest the best possible training and job offers. The web service will map its matching metadata scheme to each Agency's database fields. Several other Grid/Web services will offer information on jobs and training programs directly from the organizations which offer these programs. In this way, the civil servants will only be concerned with their consulting services while information on vocational training and job offers will come directly from the system.

The Grid can be defined as a layer of secure networked services that allow users single sign-on access to a distributed collection of data resources. Each user interested in connecting to a Grid/web service must have an issued certificate and all data transferred through the Grid can be encrypted. This architecture, as it will deal with personal data that must be secured from unauthorized access will be based on the Open Grid Services Architecture (OGSA) that defines standard mechanisms for creating, naming, and discovering transient Grid service instances.

The paper is structured in the following sections: Section 2 presents a theoretical overview of the related literature. Section 3 describes the technology of the proposed service and the proposed solution. Section 4 presents the results while Section 5 is devoted to discussion and future works.

\section{RELATED WORK}

Several applications exist that have being developed in the framework of EU funded programs (EQUAL $1 \& 2$, eTen, etc) or by private initiatives, to support civil servants in their work. The limitation in all these applications is that they are tailor-build to the needs of each agency and the civil servants have to insert in these applications all the information about the programs of training institutions and job offers that they find, in order to match it with their beneficiaries' needs. The proposed solution is not aiming to replace these 
applications but to interconnect them in a wider secure knowledge grid offering added value services to all involved.

The innovation of the proposed system is its distributed nature based on the GRID/Web services technology that offers a fast, reliable and effective communication between involved parties, providing at the same time up-to-date information customized each time to the needs of the beneficiaries and vise versa, minimizing at the same time communication expenses, data replication and expiration.

\section{METHODOLOGY}

Grid technologies support the sharing and coordinated use of diverse resources in dynamic "virtual organizations" (VOs) - that is, the creation, from geographically and organizationally distributed components, of virtual computing systems that are sufficiently integrated to deliver desired Quality of Service (QoS). The Grid idea emerged initially as a model that combines the resources of many separate computers connected by a network (usually the internet) to solve large-scale computation problems. Web services, as a new, interoperable and effective way to provide functionality over the internet, drew attention to the Grid technology, and especially to Grids build on web services (GRID $\backslash$ WEB services). Following this approach a Grid can be defined as a layer of networked services that allow users single sign-on access to a distributed collection of compute, data and application resources. The above lead to the development of the Open Grid Service Architecture that defines standard mechanisms for creating, naming, and discovering transient Grid service instances, provides location transparency and multiple protocol bindings for service instances and supports integration with underlying native platform facilities. The Open Grid Services Architecture also defines, in terms of Web Services Description Language (WSDL) interfaces and associated conventions, mechanisms required for creating and composing sophisticated distributed systems, including lifetime management, change management, and notification.

This work presents the effort to exploit this innovative architecture so that added value services are offered to the area of civil services. Tools for civil servants (Karadimas and Papastamatiou 2008) are to be developed in a distributed, interoperable and secure infrastructure, namely the Grid infrastructure. Thus the integration of remotely located, disjoint and diverse processing and data storage facilities under a common software architecture will be ensured, and moreover, due to the advanced security mechanism provided by the infrastructure, authentication, authorization and policy management will be facilitated, providing users with the possibility to form Virtual Organizations and share data between them.

The platform on which the Grid/Web services will be built is the open source Globus ${ }^{\circledR}$ Toolkit. Globus ${ }^{\circledR}$
Toolkit is a fundamental enabling technology for the "Grid," letting people share computing power, databases, and other tools securely online across corporate, institutional, and geographic boundaries without sacrificing local autonomy. The toolkit includes software for security, information infrastructure, resource management, data management, communication, fault detection, and portability. It is packaged as a set of components that will be used for the development of the proposed Grid/web services.

The proposed system is a great opportunity to upgrade the role and efficiency of civil services. It is a cost effective and reliable way to offer accurate and up-todate information on vocational training courses and job vacancies in the market. It is also a great opportunity for the training organizations to promote their courses and provide training relevant to the real needs of the jobmarket.

Civil servants have to deal with unemployed people from underprivileged citizen groups who face several problems (lack of skills, disabilities, language problems, family problems,etc). Their efforts focus on, first of all, identifying their problems and then helping them to overcome them. Apart from empowering and supporting individuals to overcome their personal problems, they also try to find for them suitable training programs and jobs.

To be effective, they have to make frequent contacts with training institutions and employment agencies, or directly with employers in order to locate job vacancies and training programs. The problem they face is that they have access to a limited number of resources as many as they can afford to contact or find. This is the reason why their work is focussed more on the empowering part and less in the actually finding of a job for these individuals. It is easier for them to recommend training in one of the training organizations that cooperate with their agencies, and to prepare them for a potential job interview, helping them at the same time to identify what is the appropriate line of work for each individual. Training, however is not directly connected to employment, and most of the courses offered are on basic skills, like communication skills, business English, Information Technology, marketing, etc.

The proposed architecture can solve all those problems that civil servants are facing today by identifing the most reliable and up-to-date sources of information through searching several different on line databases.

The integration of the web services offering access to vocational training courses and job offers is accomplished through the described Grid/Web services architecture. The web services, constituting the GRID, will be custom-made for civil servants needs, performing a specific set of operations that will offer up-to-date information on available Vocational training courses and job offers from different organizations upon request from the civil servants and customized to the beneficiaries' needs. 
A Broker (machine that will host the Universal Description, Discovery and Integration (UDDI) directory) is required in order to setup this architecture where all these web services under the GRID will be registered setting up a private UDDI registry.

A web service (W3C Working Group Note 2004) must be register to a UDDI directory in order to be published and reveal its functionality to the world. UDDI is an XML-based (World Wide Web Consortium 2006) standard for describing, publishing, and finding Web services. Service consumers can then require from the broker to locate a required web service and use it to implement a business solution. Each web service, located at the web server of a social agency, will act as one of those service consumers and will use all the web services register to the UDDI registry.

Setting up the "social Grid", specific WSDL (GRIDWSDL) should be developed for each web service to comply to. When each web service is discovered, the GRID-WSDL XML-format protocol will help other systems that need its functionality to understand the way that they can interface it. WSDL describes how to access a web service and not the business logic of this web service.

To utilize the web services functionality a common language between these web services should be defined. XML/SOAP messages will be used for exchanging information. SOAP is a lightweight protocol intended for exchanging structured information in a decentralized, distributed environment (Gudgin et al. 2003).

$\mathrm{XML}$ is the technology used with SOAP messages that form the requests and responses from a web service. A client invokes a web service by sending an XML/SOAP message, and then waits for a corresponding $\mathrm{XML} / \mathrm{SOAP}$ response. Because all communication is in $\mathrm{XML}$, web services are not tied to any one operating system or programming language. The content of these messages will comply with the structure presented in (Karadimas and Papastamatiou 2008) addressing a common approach compliant with the EURES system (The European Job Mobility Portal).

Finally, an application that from a single point will give access to all this information from the registered web services will be developed. The application will enclose a smart search engine that will communicate with the web services subscribed to the UDDI registry and request information according to the beneficiaries needs. The results will be presented to the civil servant along with more information of the organization offering them. All the nodes described on the next diagram communicate with each other through a Transmission Control Protocol/Internet Protocol (TCP/IP).

The advantage of the described architecture is that the pool of available vocational training courses and job offers will grow rapidly as each new organization that desires to join the "social Grid" needs to follow some simple steps in order to develop and register its own web service to the GRID's UDDI. Then the web service will offer suggestions upon requested information for the organization's courses or job offers.

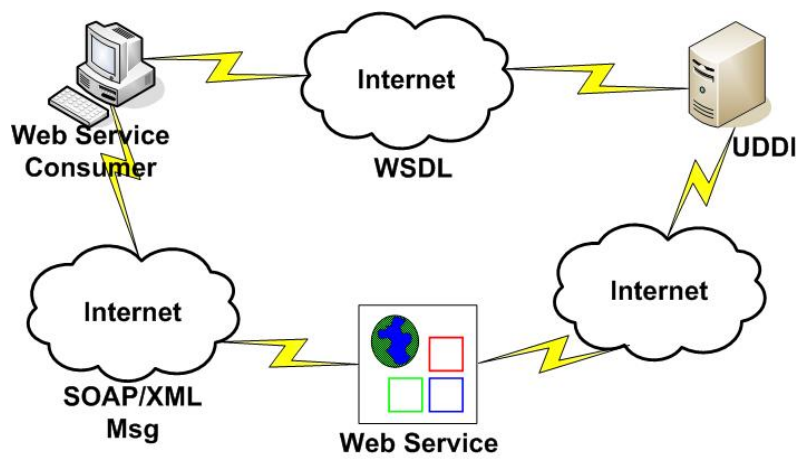

Figure 1: Description of the proposed architecture

Administration costs for the "social Grid" are minimized since the administrator's tasks are limited to observing the successful integration of the new web services to the Grid.

\section{RESULTS}

The proposed solution provides civil servants with a decision support tool with access to a tank of educational programs and job offers otherwise difficult to find and filter.

The Grid/Web services solution for social services is a great opportunity to upgrade the role and efficiency of civil servants. It is a cost effective and reliable way to offer accurate and up-to-date information on vocational training courses and job offers. It is also a great chance for the training organizations to promote their courses and provide training relevant to the real needs of the jobmarket. The proposed architecture is easy to implement, open to the integration of further web services of organizations offering vocational training and job offers, and easy to maintain and extend.

\section{DISCUSSION AND FUTURE WORK}

The proposed solution and especially its GRID/Web services architecture, under a common metadata scheme can find implementation in many other sectors, like ehealth, e-commerce, the tourism sector, etc. Generally, there is a great need for secure networks for the exchange of data stored in heterogeneous sources. Above such platforms, several other applications can be developed that will make use of the data exchanged through these grid/web services. Other fields of application are the inter-exchange of information between large companies with many branches, public authorities, schools, universities, the military, etc.

\section{REFERENCES}

EURES: The European Job Mobility Portal. http://europa.eu.int/eures (Accessed on February 10, 2008).

Gudgin M., Hadley M., Mendelsohn N., Moreau J-J. and Frystyk Nielsen H. 2003. "SOAP Version 1.2 Part1: 
Messaging Framework", W3C Recommendation, http://www.w3.org/TR/soap12-part1 Accessed on February 23, 2008)

Karadimas N.V. and Papastamatiou N.P. 2008. "Inquiring Training and Employment Offers on the Web Using Web Services". 7th World Scientific and Engineering Academy and Society, International Conference on Applied COmputational Science (ACOS '08), Hangzhou, China, 68 April, [In Press, Corrected proof].

W3C Working Group Note 2004. "Web Services Architecture". http://www.w3.org/TR/2004/NOTE-wsarch-20040211/. (Accessed on February 10, 2007).

World Wide Web Consortium (W3C). 2006. "eXtensible Markup Language (XML)”. http://www.w3.org/xml (Accessed on February 10, 2007).

\section{AUTHOR BIOGRAPHIES}

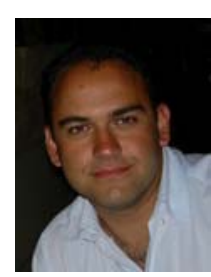

NIKOLAOS V. KARADIMAS was born in Athens, Greece and he graduated from the Techonogical Educational Institute (T.E.I.) of Patra in 1995 with a Bachelor's degree in Electrical Engineering. He then received a Bachelor's degree with Honours in Electronic Engineering and a Masters degree in Computer Science from Glasgow Caledonian University, Scotland in 1997 and 1998, respectively. He also holds a Masters degree in Distributed and Multimedia Information Systems (1999) from HeriotWatt University, Scotland and a PhD degree (2007) from the National Technical University of Athens. Since 2007, he is a post-doctoral researcher at the Multimedia Technology Laboratory within National Technical University of Athens and lecturer (407/80) at the Hellenic Military Academy, as well. He is also teaching in Technical NCO Academy and in T.E.I. of Piraeus. He is a Chartered Engineer, member of the Greek Chamber of Engineers, member of IEEE and member of IET. His research interests are in the fields of Databases, Optimization Techniques, Geographical Information Systems, Decision Support Systems and Multimedia.

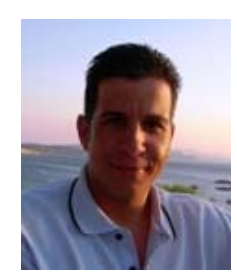

NIKOLAOS P. PAPASTAMATIOU was born in Athens, Greece and he graduated from Aegean University, Samos in 1998 with a Bachelor's degree on Mathematics. He then received a Masters degree in Distributed and Multimedia Information Systems from Heriot-Watt University, Scotland in 1999. From 2002 he is working for a software house as a web analyst/developer. He has followed several seminars on web technologies (XML Web services, Programming with XML in the .Net Framework, ASP.NET) and he has participated to many European and National research projects. Currently he is following a vocational training course on project management from National and Kapodistrian University of Athens. His research interests are in the fields of Web Development, Databases, Web Services, Grid Computing, Natural Language Processing and Multimedia Applications.

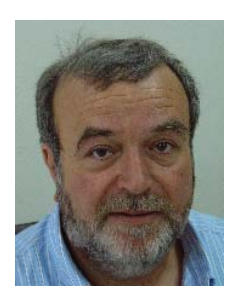

VASSILI LOUMOS received the Diploma of Electrical Engineering and the $\mathrm{PhD}$ degree in Computer Science from the National Technical University of Athens (NTUA), Greece in 1975 and 1989. From 1975 to 1978 he was a research engineer at the Centre National de Recherhe Scientifique, Paris, France. From 1980 to 1982 he was with CGEE ALSHTOM as a field engineer. In 1990 he joined the faculty of the School of Electrical \& Computer Engineering of the NTUA where he is presently a professor. He is teaching Multimedia Technologies and Computer Graphics. His research interests are in the fields of Multimedia, Computer Vision and Internet Navigation. 